\title{
ZEROS OF STIELTJES AND VAN VLECK POLYNOMIALS BY
}

\author{
MAHFOOZ ALAM ${ }^{1}$
}

\begin{abstract}
The study of the polynomial solutions of the generalized Lamé differential equation gives rise to Stieltjes and Van Vleck polynomials. Marden has, under quite general conditions, established varied generalizations of the results proved earlier by Stieltjes, Van Vleck, Bôcher, Klein, and, Pólya, concerning the location of the zeros of such polynomials. We study the corresponding problem for yet another form of the generalized Lamé differential equation and generalize some recent results due to Zaheer and to Alam. Furthermore, applications of our results to the standard form of this differential equation immediately furnish the corresponding theorems of Marden. Consequently, our main theorem of this paper may be considered as the most general result obtained thus far in this direction.
\end{abstract}

1. Introduction. There exist [2] at most $C(n+p-2, p-2)$ polynomials $V(z)$ such that for $\Phi(z)=V(z)$ the generalized Lamé differential equation

$$
\frac{d^{2} w}{d z^{2}}+\left[\sum_{j=1}^{p} \frac{\alpha_{j}}{\left(z-a_{j}\right)}\right] \cdot \frac{d w}{d z}+\frac{\Phi(z)}{\prod_{j=1}^{P}\left(z-a_{j}\right)} \cdot w=0,
$$

where $\Phi(z)$ is a polynomial of degree at most $(p-2)$ and $\alpha_{j}, a_{j}$ are complex constants, has a polynomial solution $S(z)$ of degree $n$. Each polynomial $V(z)$ and the corresponding polynomial $S(z)$ are called [5, pp. 36-37] a Van Vleck polynomial and a Stieltjes polynomial, respectively (Marden [6, p. 934] calls $V(z)$ the characteristic polynomial).

We shall investigate the location of the zeros of the system of polynomials that arise in the study of the polynomial solutions of the differential equation

$$
\begin{array}{r}
\frac{d^{2} w}{d z^{2}}+\left[\sum_{j=1}^{p} \alpha_{j}\left\{\prod_{t=1}^{n_{j}-1}\left(z-b_{j t}\right) / \prod_{s=1}^{n_{j}}\left(z-a_{j s}\right)\right\}\right] \cdot \frac{d w}{d z} \\
+\left[\frac{\Phi(z)}{\prod_{j=1}^{p} \Pi_{s=1}^{n_{j}}\left(z-a_{j s}\right)}\right] \cdot w=0,
\end{array}
$$

Received by the editors September 8, 1977 and, in revised form, June 19, 1978.

AMS (MOS) subject classifications (1970). Primary 30A08; Secondary 33A70.

Key words and phrases. Generalized Lamé differential equations, Stieltjes polynomials, Van Vleck polynomials, and covering functions.

'The results in this paper are partly contained in the author's doctoral thesis (1977) at Aligarh Muslim University, Aligarh, under the supervision of Dr. Neyamat Zaheer. The author wishes to thank him for his useful suggestions and advice all along. This work was performed under the research fellowship U.G.C. (Government of India). 
where $\Phi(z)$ is a polynomial of degree at most $\left(n_{1}+n_{2}+\cdots+n_{p}-2\right)$ and $a_{j s}, b_{j t}$ and $\alpha_{j}$ are suitable complex constants. It is easy to note that the differential equation (1.2) can be written in the form (1.1) and, further, that (1.2) is of the form (1.1) in case $n_{j}=1$ for all $j$.

The study of the zeros of the polynomials $S(z)$ and $V(z)$ in relation to the differential equation (1.1) has extensively been made by many mathematicians (see Marden [6, pp. 935-936]) under suitable conditions on the singularities $a_{j}$, but only for positive real values of the constants $\alpha_{j}$. Marden [6], for the first time gave the treatment of (1.1) subject to the condition $\left|\arg \alpha_{j}\right| \leqslant \gamma<\pi / 2$ and obtained varied generalizations of the results (see [6, Theorems 1(a) - 2(a)]) established earlier by Stieltjes [8], Van Vleck [10], Bôcher [1], Klein [3] and Pólya [7]. In this paper we intend to study the zeros of the polynomials $S(z)$ and $V(z)$ in relation to the differential equation (1.2). The results that we obtain are valid for both the forms (1.1) and (1.2), whereas the corresponding known results [6] apply only to the form (1.1) and turn out as corollaries to our results. We shall, however, make strong use of the concepts of covering functions as introduced by Marden [6].

In our attempt to generalize a result due to Marden [6, Theorem 4], we have in a recent paper (co-authored with Zaheer [11]) obtained some results for the first time in relation to the differential equation (1.2). The following result [11, Corollary (2.5)] which is a special consequence of our much more general theorem [11, Theorem (2.3)], plays an important role in the proof of our main theorem in the next section.

THEOREM (1.1). If $\left|\arg \alpha_{j}\right| \leqslant \gamma<\pi / 2$ for every $j=1,2, \ldots, p$ and if all the points $a_{j s}, b_{j t}$ (occurring in the differential equation (1.2)) lie on or inside the circle $C:|z-c|=a$, then the zeros of each Stieltjes polynomial and the zeros of each Van Vleck polynomial, associated with the differential equation (1.2), lie in the circular region

$$
|z-c| \leqslant a \sec [\{(q-1) \pi+\gamma\} /(2 q-1)]
$$

where $q=\max \left\{n_{1}, n_{2}, \ldots, n_{p}\right\}$.

As remarked in [11] Theorem 1.1 implies for $q=1$ a result of Marden [6, Theorems 4 and 5, Theorem (9.1)] that if all $a_{j}$ of equation (1.1) lie in $|z-c| \leqslant a$, then all the zeros of the Stieltjes and Van Vleck polynomials lie in $|z-c| \leqslant a \sec \gamma$ (where it is assumed that, for all $j,\left|\arg \alpha_{j}\right| \leqslant \gamma<\pi / 2$ ). We note however that if we take a case of equation (1.2) with $q>1$ and bring it to the form (1.1) then, in general, Theorem 1.1 (about equation (1.2)) and Marden's result (about equation (1.1)) are independent. To see this consider the equation

$$
w^{\prime \prime}(z)+\alpha \frac{z-i}{z^{2}-1} w^{\prime}(z)+\frac{k}{z^{2}-1} w=0
$$


(where the constant $k=k(n)$ is so chosen that $\left(1.2^{\prime}\right)$ has a polynomial solution $S(z)$ of degree $n)$. Here $p=1, n_{1}=2, b=i, a_{1}=1, a_{2}=-1$, so all the $a$ 's and $b$ 's lie in $|z| \leqslant 1$ and if we assume that $|\arg \alpha|<\gamma<\pi / 2$, then it follows from Theorem 1.1 (as $q=n_{1}=2$ ) that all the zeros of $S(z)$ lie in $|z| \leqslant \sec [(\pi+\gamma) / 3]$. On the other hand, we may bring $\left(1.2^{\prime}\right)$ into the form

$$
w^{\prime \prime}(z)+\left[\frac{\alpha_{1}}{z-1}+\frac{\alpha_{2}}{z+1}\right] w^{\prime}(z)+\frac{k}{z^{2}-1} w=0,
$$

where now $2 \alpha_{1}=\alpha(1-i), 2 \alpha_{2}=\alpha(1+i)$ and hence $\left|\arg \alpha_{j}\right|<\gamma+(\pi / 4)$, $j=1,2$. So if we assume that $\gamma<\pi / 4$, then it follows from Marden's result that all the zeros of $S(z)$ lie in $|z| \leqslant \sec [\gamma+(\pi / 4)]$. Now if $0<\gamma<\pi / 8$, then $\gamma+(\pi / 4)<(\pi+\gamma) / 3$, so Marden's result is for these $\gamma$ better than Theorem 1.1, while for $\pi / 8<\gamma<\pi / 4$ Theorem 1.1 yields the smaller disk, and finally for $\pi / 4<\gamma<\pi / 2$ only Theorem 1.1 can be applied.

The following results [11, Lemmas (2.1) and (2.2)] express the zeros of Stieltjes and Van Vleck polynomials as roots of certain equations.

Proposition (1.2). Every zero $z_{k}$ of an nth degree Stieltjes polynomial $S(z)$ in relation to (1.2) is either one of the points $a_{j s}\left(1<j<s ; 1<s<n_{j}\right)$ or satisfies the equation

$$
\frac{1}{2}\left[\sum_{j=1}^{p} \alpha_{j}\left\{\prod_{t=1}^{n_{j}-1}\left(z_{k}-b_{j t}\right) / \prod_{s=1}^{n_{j}}\left(z_{j}-a_{j s}\right)\right\}\right]+\sum_{j \neq k, j=1}^{n} \frac{1}{z_{k}-z_{j}}=0
$$

with the convention that $\prod_{i=1}^{n_{j}-1}\left(z-b_{j t}\right) \equiv 1$ when $n_{j}=1$.

Proposition (1.3). Every zero $t_{k}$ of a Van Vleck polynomial $V(z)$ in relation to (1.2), if not an $a_{j s}$, is either one of the zeros of $S^{\prime}(z)$ or satisfies the equation

$$
\left[\sum_{j=1}^{p} \alpha_{j}\left\{\prod_{t=1}^{n_{j}-1}\left(t_{k}-b_{j t}\right) / \prod_{s=1}^{n_{j}}\left(t_{k}-a_{j s}\right)\right\}\right]+\sum_{j=1}^{n-1} \frac{1}{t_{k}-z_{j}^{\prime}}=0,
$$

where $S(z)$ is an nth degree Stieltjes polynomial corresponding to $V(z)$ and the $z_{j}^{\prime}$ are the zeros of $S^{\prime}(z)$.

For $q=1$, equations (1.3) and (1.4) reduce, respectively, to equations (S) and $(F)$ as given in Marden [6, p. 935].

In respect to the differential equation (1.1), Marden obtained quite a general result [6, Theorem 5] for arbitrary convex regions by introducing the concept of covering functions $[6, \S 3]$. By the covering function of an arbitrary convex region $K$ we mean a function $k(\lambda)$ such that the inequality

$$
|z-\lambda| \leqslant k(\lambda)
$$

holds for all values of $\lambda$ by and only by the points $z$ of $K$. We know that one covering function $k(\lambda)$ of a given convex region $K$ may always be chosen as 
the maximum value of $|z-\lambda|$ for points $z$ in $K$. We also know [6] that every covering function of a convex region is always nonnegative and that if $k(\lambda)$ is any real nonnegative function, the totality of points $z$ which (for all values of $\lambda$ ) satisfy the inequality (1.5), form a convex region $K$ with $k(\lambda)$ as a covering function.

2. Main results. In this section, we shall obtain results concerning the zeros of Stieltjes and Van Vleck polynomials in respect to the differential equation (1.2) and for arbitrary convex regions. Our main theorem in this paper is the most general result known thus far in this direction and deduces as corollaries the previously known results due to Zaheer [12, Theorems (2.1) and (2.2)], Zaheer and Alam [11, Corollary (2.5)], concerning the differential equation (1.2), and also the results due to Marden [6, Theorems 1(a)-6(b)] concerning the differential equation (1.1). Our main result is the following.

THEOREM (2.1). If $\left|\arg \alpha_{j}\right| \leqslant \gamma<\pi / 2$ and if all the points $a_{j s}$, $b_{j t}$ (occurring in the differential equation (1.2)) lie in a convex region $K$ with $k(\lambda)$ as covering function, then the zeros of each Stieltjes polynomial and the zeros of each Van Vleck polynomial, associated with the differential equation (1.2), lie in the convex region $K^{\prime}$ which contains $K$ and which has as covering function

$$
k^{\prime}(\lambda)=k(\lambda) \sec [\{(q-1) \pi+\gamma\} /(2 q-1)]
$$

where $q=\max \left\{n_{1}, n_{2}, \ldots, n_{p}\right\}$.

PRoof. Since all the points $a_{j s}, b_{j t}\left(1 \leqslant s \leqslant n_{j}, 1 \leqslant t \leqslant n_{j}-1,1 \leqslant j \leqslant p\right)$ lie in the convex region $K$ with $k(\lambda)$ as covering function, we see that for each value of $\lambda$ the points $a_{j s}, b_{j t}$ would, therefore lie in the circular region $|z-\lambda| \leqslant k(\lambda)$. From Theorem (1.1), we now infer that the zeros of each Stieltjes polynomial $S(z)$ and the zeros of each Van Vleck polynomial $V(z)$ lie in the circular region

$$
|z-\lambda| \leqslant k(\lambda) \sec [\{(q-1) \pi+\gamma\} /(2 q-1)]
$$

for each value of $\lambda$. This means that the zeros under consideration lie in a convex region $K^{\prime}$ which has

$$
k^{\prime}(\lambda)=k(\lambda) \sec [\{(q-1) \pi+\gamma\} /(2 q-1)]
$$

as a covering function.

Further, we know [6] that if a convex region $K$ has $k(\lambda)$ as a covering function and another convex region $K^{\prime}$ has $k^{\prime}(\lambda)$ as a covering function such that $k^{\prime}(\lambda) \geqslant k(\lambda)$, then $k^{\prime} \supseteq K$. Now, since $0 \leqslant \gamma<\pi / 2$, we easily see that

$$
0 \leqslant\{(q-1) \pi+\gamma\} /(2 q-1)<\pi / 2,
$$

and, hence, that $k^{\prime}(\lambda) \geqslant k(\lambda)$. This implies that $K^{\prime}$ encloses $K$ and our proof is complete. 
As an immediate consequence of Theorem (2.1), we obtain the following result due to Marden [6, Theorem 5].

COROLlaRY (2.2). If all the points $a_{j}$ in relation to differential equation (1.1) lie in a convex region $K$ with $K(\lambda)$ as covering function, then the zeros of each Stieltjes polynomial and the zeros of each Van Vleck polynomial, associated with differential equation (1.1), lie in a convex region $K^{\prime}$ which contains $K$ and which has as covering function $k^{\prime}(\lambda)=k(\lambda) \sec \gamma$.

Proof. In Theorem (2.1), if we take all $n_{j}$ 's to be 1 (i.e., $q=1$ ), differential equation (1.2) reduces to differential equation (1.1), with the constants $a_{j}$ corresponding to the constants $a_{j 1}$, and Corollary (2.2) is fairly obvious.

Making use of the covering function for circles, as employed by Marden [6, p. 938], Theorem (1.1) may easily be deduced from Theorem (2.1), and, hence, Theorem (2.1) may be regarded as an extension of Theorem (1.1).

COROllaRY (2.3). If $\left|\arg \alpha_{j}\right| \leqslant \gamma<\pi / 2$ and if all the points $a_{j s}$, $b_{j t}$ (cf. (1.2)) lie in or on a given ellipse $E$ with major axis 2a, the zeros of each Stieltjes polynomial and the zeros of each Van Vleck polynomial (associated with (1.2)) lie in or on the confocal ellipse $E^{\prime}$ with major axis

$$
2 a \sec [\{(q-1) \pi+\gamma\} /(2 q-1)],
$$

where $q=\max \left\{n_{1}, n_{2}, \ldots, n_{p}\right\}$.

Proof. First, we give the proof for the special case when the ellipse $E$ has its centre, vertices, and foci at the points $(0,0),( \pm a, 0)$, and $( \pm c, 0)$, respectively. Then (cf. [6, p. 939]) one covering function of $E$ is given by

$$
k(\lambda)= \begin{cases}a\left(1+c^{-2}|\lambda|^{2}\right)^{1 / 2} & \text { if } \operatorname{Re}(\lambda)=0 \\ \infty & \text { if } \operatorname{Re}(\lambda) \neq 0\end{cases}
$$

(The equation corresponding to (2.1), as stated in Marden [6], has an obvious misprint and $\lambda$ should be replaced in his equation by $|\lambda|$.) Now the covering function of the convex region $K^{\prime}$ of Theorem (2.1) with $K=E$ is given by

$$
K^{\prime}(\lambda)= \begin{cases}a\left(1+c^{-2}|\lambda|^{2}\right)^{1 / 2} \sec [\{(q-1) \pi+\gamma\} /(2 q-1)] & \text { if } \operatorname{Re}(\lambda)=0 \\ \infty & \text { if } \operatorname{Re}(\lambda) \neq 0\end{cases}
$$

From Theorem (2.1) we, therefore, infer that $K^{\prime}$ is precisely the ellipse $E^{\prime}$ and our corollary follows in the case under consideration. The general case, however, follows from similar arguments as in Marden (cf. the discussion preceding Theorem 6a [6, p. 939], with equations $(S)$ and $(F)$ replaced in his arguments by equations (1.3) and (1.4), respectively). 
In Corollary (2.3) if we take all $n_{j}$ 's to be 1 (i.e., $q=1$ ), it is precisely a result due to Marden [6, Theorem 6a].

Since a line segment with $c_{1}, c_{2}$ as end points is a limiting case of an ellipse (i.e. it is the intersection of all confocal ellipses having common foci at $c_{1}$ and $c_{2}$ ), we obtain from Corollary (2.3) the following result due to Zaheer [12, Theorems (2.1) and (2.2)] in relation to differential equation (1.2).

COROLlaRY (2.4). If $\left|\arg \alpha_{j}\right| \leqslant \gamma<\pi / 2$ and if all the points $a_{j s}, b_{j t}$ (cf. (1.2)) lie on the line segment joining the points $c_{1}$ and $c_{2}$, then the zeros of each Stieltjes polynomial and the zeros of each Van Vleck polynomial (associated with (1.2)) lie in the region $K$ given by

$$
K=\left\{z:\left|z-c_{1}\right|+\left|z-c_{2}\right| \leqslant\left|c_{1}-c_{2}\right| \sec [\{(q-1) \pi+\gamma\} /(2 q-1)]\right\},
$$

where $q=\max \left\{n_{1}, n_{2}, \ldots, n_{p}\right\}$.

We note that the above corollary for $q=1$ is essentially a result due to Marden [6, Theorem 6b].

3. Final remarks. As we have seen in previous sections, various mathematicians had obtained different results for special convex regions from time to time, in relation to differential equation (1.1), till Marden gave his general theorem for arbitrary convex regions [6, Theorem 5]. Also, in relation to differential equation (1.2), some results for special convex regions such as for circles [11, Corollary (2.5)], and for line segments [12, Theorems (2.1) and (2.2)] have already been obtained, but our Theorem (2.1) now gives the general result.

We also note that the part of our Theorem (2.1) that concerns Stieltjes polynomials may be regarded as a generalization of Lucas' theorem $\left[5\right.$, Theorems $\left.(6,1),(6,1)^{\prime},(6,2)\right]$ or $[4]$ to systems of partial fraction sums.

Finally, we remark that our results are applicable to certain classical orthogonal polynomials [9, pp. 29, 58-63, 74, 155], e.g. Legendre, Jacobi, Tchebichef, and ultraspherical polynomials of first or second kind which are special cases of Stieltjes polynomials (when $p=2, n_{1}=n_{2}=1=q ; \alpha_{1}, \alpha_{2}>$ $0 ; a_{11}=-a_{21}=1$ in equation (1.2)). Our Theorem (2.1) immediately implies the well-known result $[5$, p. 41] [9, p. 44] that the zeros of such polynomials lie in the interval $[-1,1]$, in view of the fact that one covering function of the closed interval $[-a, a]$ is given by

$$
k(\lambda)= \begin{cases}|\lambda-a| & \text { if } \operatorname{Re}(\lambda)=0 \\ \infty & \text { if } \operatorname{Re}(\lambda) \neq 0\end{cases}
$$

The location of the zeros of more general forms (i.e. when $\alpha_{1}, \alpha_{2}$ are complex numbers, in general) of such polynomials is much more involved and cumbersome to obtain via the existing methods available in the theory of 
classical orthogonal polynomials. Our results, however, have an easy access to the location of the zeros of the general forms of these polynomials. A typical example to this effect is the following new result concerning the zeros of the generalized Jacobi polynomials.

THEOREM (3.1). If $\operatorname{Re}(\alpha)>-1, \operatorname{Re}(\beta)>-1$, and $\gamma=\max \{|\arg (\alpha+1)|$, $|\arg (\beta+1)|\}$, then the zeros of every nth degree generalized Jacobi polynomial $P_{n}^{(\alpha, \beta)}(z)$ lie in the region $K_{\alpha, \beta}$ given by

$$
K_{\alpha, \beta}=\{z:|z-1|+|z+1| \leqslant 2 \sec \gamma\} .
$$

Proof. The generalized Jacobi polynomials $P_{n}^{(\alpha, \beta)}(z)$ are polynomial solutions of the differential equation

$$
\begin{array}{r}
\left(z^{2}-1\right) \cdot \frac{d^{2} w}{d z^{2}}+[(\alpha-\beta)+(\alpha+\beta+2) z] \frac{d w}{d z} \\
-n(n+\alpha+\beta+1) \cdot w=0
\end{array}
$$

which is a special case of differential equation (1.2) for $q=1 ; p=2$; $a_{11}=-a_{21}=1 ; \alpha_{1}=\alpha+1, \alpha_{2}=\beta+1(\alpha, \beta$ being arbitrary complex numbers). Consequently, the generalized Jacobi polynomials $P_{n}^{(\alpha, \beta)}(z)$ are the Stieltjes polynomials. The hypotheses on $\alpha, \beta$ imply that $\left|\arg \alpha_{j}\right| \leqslant \gamma<\pi / 2$ for $j=1,2$. In view of (3.1), our Theorem (2.1) or Marden's result [6, Theorem 5] says that all the zeros of $P_{n}^{(\alpha, \beta)}(z)$ lie in the region $K^{\prime}$ whose one covering function is given by

$$
k^{\prime}(\lambda)= \begin{cases}|\lambda-1| \sec \gamma & \text { if } \operatorname{Re}(\lambda)=0 \\ \infty & \text { if } \operatorname{Re}(\lambda) \neq 0\end{cases}
$$

Or, equivalently,

$$
k^{\prime}(\lambda)= \begin{cases}\left(1+|\lambda|^{2}\right)^{1 / 2} \sec \gamma & \text { if } \operatorname{Re}(\lambda)=0 \\ \infty & \text { if } \operatorname{Re}(\lambda) \neq 0\end{cases}
$$

This region $K^{\prime}$ is thus seen to be an ellipse (cf. (2.1)) with center $(0,0)$, vertices $( \pm \sec \gamma, 0)$ and foci $( \pm 1,0)$. Therefore, $K^{\prime}$ is precisely the region $K_{\alpha, \beta}$ as claimed in the theorem.

ACKNOWLEDGEMENT. The author is thankful to the referee who suggested to include equations $\left(1.1^{\prime}\right)$ and $\left(1.2^{\prime}\right)$.

\section{REFERENCES}

1. M. Bôcher, Über die Reihenentwickelungen der Potentialtheoreie, Teubner, Leipzig, 1894, pp. 215-218.

2. E. Heine, Handbuch der Kugelfunctionen, Bd. I (2nd ed.), G. Reimer, Berlin, 1878, pp. $472-476$. 
3. F. Klein, Über lineare Differentialgleichungen der zweiten Ordnung, Göttingen, 1894, pp. 211-218.

4. F. Lucas, Propriétés géométriques des fractions rationnelles, C. R. Acad. Sci. Paris 77 (1874), 431-433; ibid. 78 (1874), 140-144; ibid. 78 (1874), 180-183; ibid. 78 (1874), 271-274.

5. M. Marden, Geometry of polynomials, Math. Surveys, no. 3, Amer. Math. Soc., Providence, R. I., 1966.

6. __ On Stieltjes polynomials, Trans. Amer. Math. Soc. 33 (1931), 934-944.

7. G. Pólya, Sur un théorème de Stieltjes, C. R. Acad. Sci. Paris 155 (1912), 767-769.

8. T. J. Stieltjes, Sur certain polynômes qui verifient une équation différentielle, Acta Math. 6-7 (1885), 321-326.

9. G. Szegö, Orthogonal polynomials, Amer. Math. Soc. Colloq. Publ., vol. 23, Amer. Math. Soc., Providence, R. I., 1967.

10. E. B. Van Vleck, On the polynomials of Stieltjes, Bull. Amer. Math. Soc. 4 (1898), 426-438.

11. N. Zaheer and M. Alam, On the zeros of Stieltjes and Van Vleck polynomials, Trans. Amer. Math. Soc. 229 (1977), 279-288.

12. N. Zaheer, On Stieltjes and Van Vleck polynomials, Proc. Amer. Math. Soc. 60 (1976), 169-174.

Department of Mathematics, Aligarh Muslm University, Aujgarh 202001, India 